\title{
Integrated models of care for diabetes and hypertension in low- and middle-income countries (LMICs) : Protocol for a systematic review
}

Jeannine Uwimana Nicol ${ }^{1,2^{*}}$ D, Anke Rohwer ${ }^{1}$, Taryn Young ${ }^{1}$, Charlotte M Bavuma ${ }^{3}$ and Joerg J Meerpohl ${ }^{4}$

\begin{abstract}
Background: In low- and middle-income countries (LMICs), the burden of non-communicable diseases (NCDs) is growing against an existing burden of other diseases such as HIV/AIDS. Integrated models of care can help address the rising burden of multi-morbidity. Although integration of care can occur at various levels and has been defined in numerous ways, our aim is to assess the effects of integration of service delivery at primary healthcare level in LMICs.

Methods: We will consider randomised controlled trials (RCTs), cluster RCTs, non-randomised trials, controlled beforeafter studies and interrupted time series that examine integrated models of care among people with multi-morbidities, of which diabetes or hypertension is one, living in LMICs. We will compare fully integrated models of care to standalone care, partially integrated models of care to stand-alone care and fully integrated models to partially integrated models of care. Primary outcomes include all-cause mortality, disease-specific morbidity, HbA1c, systolic blood pressure and cholesterol levels. Secondary outcomes include access to care, retention in care, adherence, continuity of care, quality of care and cost of care. We will conduct a comprehensive search in the following databases: MEDLINE, EMBASE, the Cochrane Central Register of Control Trials, LILACS, Africa-Wide Information, CINAHL and Web of Science. In addition, we will search trial registries, relevant conference abstracts and check references lists of included studies. Selection of studies, data extraction and assessment of risk of bias will be performed independently by two review authors. We will resolve discrepancies through discussion with a third author. We will contact study authors in case of missing data. If included studies are sufficiently homogenous, we will pool results in a meta-analysis. Clinical heterogeneity related to the population, intervention, outcomes and context will be documented in table format and explored through subgroup analysis. We will assess $x^{2}$ and $P^{2}$ tests for statistical heterogeneity. We will use GRADE to make judgements about the certainty of evidence and present findings in a summary of findings table.

Discussion: In light of limited evidence on the provision of comprehensive care for diabetes and hypertension, and its comorbidity in $\mathrm{LMCls}$, we believe that the findings of this systematic review will provide a synthesis of evidence on effective models of integrated care for diabetes and hypertension and their comorbidities at primary healthcare level. This will enable policy-makers to device policies and programs that are evidence informed.

(Continued on next page)
\end{abstract}

\footnotetext{
* Correspondence: jeannine@sun.ac.za

${ }^{1}$ Centre for Evidence-Based Health Care, Division of Epidemiology and Biostatistics, Department of Global Health, Faculty of Medicine and Health Sciences, Stellenbosch University, Francie van Zijl drive, Parow, Cape Town 7500 , South Africa

${ }^{2}$ School of Public Health, College of Medicine and Health Science, University of Rwanda, Kicukiro, Kigali, Rwanda

Full list of author information is available at the end of the article
}

(c) The Author(s). 2018, corrected publication January/2019. Open Access This article is distributed under the terms of the Creative Commons Attribution 4.0 International License (http://creativecommons.org/licenses/by/4.0/), which permits

unrestricted use, distribution, and reproduction in any medium, provided you give appropriate credit to the original author(s) and the source, provide a link to the Creative Commons license, and indicate if changes were made. The Creative Commons Public Domain Dedication waiver (http://creativecommons.org/publicdomain/zero/1.0/) applies to the data made available in this article, unless otherwise stated. 
(Continued from previous page)

Systematic review registration: PROSPERO CRD42018099314.

Keywords: Non-communicable diseases, Diabetes, Hypertension, Multi-morbidity, Integrated care, Low- and middleincome countries

\section{Background}

The burden of non-communicable disease (NCDs) in lowand middle-income countries (LMICs) exceeds that of high-income countries [1]. In 2015, a total of 40 million people in LMICs died of NCDs (80\% of deaths) of which 15 million deaths were premature and occurred between the ages of 30-70 years, the prime productive age of employment in most LMICs $[1,2]$. This implies that NCDs are a threat to the health of adults in LMICs, to workplace productivity and to the health of economies [3].

NCDs are an umbrella term referring to cardiovascular diseases (heart attacks and stroke), cancers, chronic respiratory diseases (such as chronic obstructive pulmonary disease and asthma) and diabetes. Most NCD deaths are caused by cardiovascular diseases (17.7 million deaths per year) followed by cancers ( 8.8 million deaths per year), respiratory diseases (3.9 million deaths per year) and diabetes (1.6 million deaths per year) [1]. NCDs account for the majority of illness in all regions of the world other than sub-Saharan Africa.

Although exposure to risk factors is the main cause of premature NCD deaths, an inadequate response to the healthcare needs of affected patients contributes a great deal [4]. Diabetes and hypertension are the major cardiovascular risks leading to target organs' damage such as the brain, heart and kidney. Currently, the number of people with diabetes is estimated to be 425 million and it is expected to increase up to 629 million in 2045, and its burden is reported to be serious in productive age [5]. Similarly, the prevalence of hypertension is increasing worldwide particularly in LMICs. According to the International Society of hypertension (ISH), around $40 \%$ of people over age of 25 years have hypertension worldwide and two third of them live in LMICs [6]. In addition to standalone burden, hypertension and diabetes co-morbidity have been reported to have a synergistic impact on bad cardiovascular disease outcomes [7].

\section{Multi-morbidities of NCDs}

Multi-morbidity is defined as the presence of two or more chronic medical conditions in an individual [8]. Multi-morbidity of NCDs poses a global healthcare challenge. In most LMICs, co-morbidity of NCDs and communicable diseases (CDs) is increasing $[9,10]$. The interaction between NCDs and CDs, particularly with human immunodeficiency virus (HIV) and its treatment with cardio-metabolic disorders, and between smoking, diabetes and tuberculosis (TB) has been highlighted in the literature $[10,11]$.

Due to improved treatment programs for HIV, people living with HIV (PLWH) are living longer with an increasing risk of developing NCDs due to direct nature of HIV infection, shared risk factors and treatment side effects of HIV [10-12].

Antiretroviral treatment (ART) of HIV infection increases the risk of hyperlipidaemia and diabetes. In addition, PLWH have a high risk of contracting NCDs through the risk associated with increasing age $[9,13]$.

Moreover in LMICs, HIV remains a major threat among vulnerable groups and particularly in some parts of Africa where HIV and AIDS are the greatest cause of life-years lost $[14,15]$.

Beside the epidemiological link between NCDs and CDs, studies in the literature highlight interaction between NCDs and psychological disorders such as depression $[16,17]$.

A study conducted in Nigeria shows that the prevalence of depression was $28 \%$ in diabetic patients and $27 \%$ in patients with hypertension [18]. In light of multi-morbidity of NCDs in particular diabetes and cardiovascular diseases, it is imperative for LMICs to have a strong and dynamic health system that can respond effectively to the growing burden of multi-morbidity.

\section{Management of NCDs}

In 2010, the WHO published a Package of Essential NCDs (PEN) interventions for primary healthcare (WHO PEN) to assist low-income countries for early detection and management of NCDs at primary healthcare (PHC) level [19]. WHO PEN, provides a package of set cost-effective interventions to be delivered at low cost and acceptable quality of care, but it does not indicate how these interventions can be delivered in an integrated manner with other chronic diseases such as HIV [19]. Hence, NCDs such as diabetes and hypertension are still managed separately from other chronic diseases such as TB and HIV [20].

In order to curtail the burden of NCDs and its co-morbidities, LMICs should consider accelerating the provision of comprehensive care and integrated strategies to improve health service delivery, efficiency and equity [13].

A paradigm shift from vertical programs towards integrated approach is required to address shared NCDs 
risks and socio-economic determinant factors [9, 21]. Haldane and colleagues argue that integrated models of care provide people with holistic options centred on health needs of people and communities and thereby enhance community self-reliance [21].

\section{Need for integrated approach for management of NCDs}

Given the multiple comorbidities of hypertension and diabetes, they require comprehensive care that is health-centred rather than disease-tailored, particularly in LMICs where both the burden of NCDs and CDs is rising [13]. In addition, LMICs need health systems that can respond more effectively and equitably to the healthcare needs of people with NCDs in order to reduce morbidity, disability and death from NCDs, and contribute to better overall health outcomes [4].

The term integration is mostly defined in the literature as "managerial or operational changes to health systems to bring together inputs, delivery, management and organization of particular service functions as means of improving coverage, access, quality, acceptability and cost-effectiveness" [22]. This may include service integrations that combine "different packages of services such as integration of service delivery points, integration at different levels of service delivery, process modifications, introduction of technologies aimed at aiding integration, and integration of management decisions" [23, 24].

Lately, most policy-makers and researchers advocate for integrated approach or programs because integrated programs increase system effectiveness and cost-effectiveness, particularly in low-resource settings $[13,25,26]$.

Curry and Ham (2012) as well as Valentijn and colleagues (2013) propose typologies of integrated care. This typology differentiates integration at micro-level as patient focused, such as case management, whereas meso-level integration focuses on groups of patients or population, and macro-level integration focuses on health systems [23, 24]. Within these levels, the typology distinguishes integration types.

We classify clinical integration as care integrated into a single process through shared guidelines and protocols across professions, and service integration as different clinical services integrated within an organisation and provided through single healthcare worker or multidisciplinary teams.

In recent years, guidelines, as well as research from LMICs (and sub-Saharan Africa in particular), have focused on integration of HIV/AIDS and TB services at service delivery level of the health system [15, 27, 28]. Drawing on these and other models of care, we have conceptualised integrated care as partial integration and full integration of service delivery as illustrated in a logic model of integrated care for diabetes and hypertension (Fig. 1). In this model, we assume that a patient has already entered the health system (i.e. through a PHC clinic and or community level) either for treatment of diabetes or hypertension, or for treatment of any other chronic disease such as HIV.

In a fully integrated model of service delivery, a patient with more than one chronic disease should receive the full package of care for all diseases at a single point of care. This "one-stop-shop" model refers to horizontal integration of primary healthcare and community health services and can involve one or more healthcare professionals. In a partially integrated model of service delivery, patients receiving treatment for one disease receive additional care related to prevention, diagnosis or treatment of another disease, but do not receive the full package of care (Fig. 1).

\section{Rationale for systematic review}

We conducted a scoping review (date of last search 8 November 2016) to assess existing evidence on the effectiveness of integrated models of care in people with diabetes or hypertension and any other comorbid disease [29]. We identified five systematic reviews, of which two examined collaborative care for diabetes and depression, two looked at collaborative care for various diseases including diabetes and depression, hypertension and diabetes, and hypertension and depression; and one focused on taskshifting and management of cardiovascular diseases and diabetes [29]. However, none of the included reviews examined collaborative care for diabetes or hypertension and communicable diseases, and only one review that assessed task-shifting included studies from LMICs.

Subsequently, another review by Haldane et al. [21] examined existing programmes that integrated healthcare delivery for diabetes, hypertension or CVDs with HIV/AIDS (date of last search October 2015). They included 17 studies of which most described existing programmes and eleven of these described programmes were in sub-Saharan Africa. Although this is useful for planning and development, there is a lack of data on the effectiveness of these programmes.

In addition, little was found on factors that facilitate or hinder integration of hypertension and diabetes with co-morbidities in LMICs. Another descriptive study from Cambodia on the management of HIV/AIDS, diabetes and hypertension showed that integration of these services was highly acceptable and led to good health outcomes [30].

A Cochrane review by Dudley and Garner assessed the effectiveness of strategies to integrate primary healthcare services in LMICs [31]. They included studies that integrated family planning into existing services; nutrition and infectious disease interventions; and sexually transmitted infections, HIV/AIDS and TB treatment. None of the included studies reported on services linked to NCDs. 


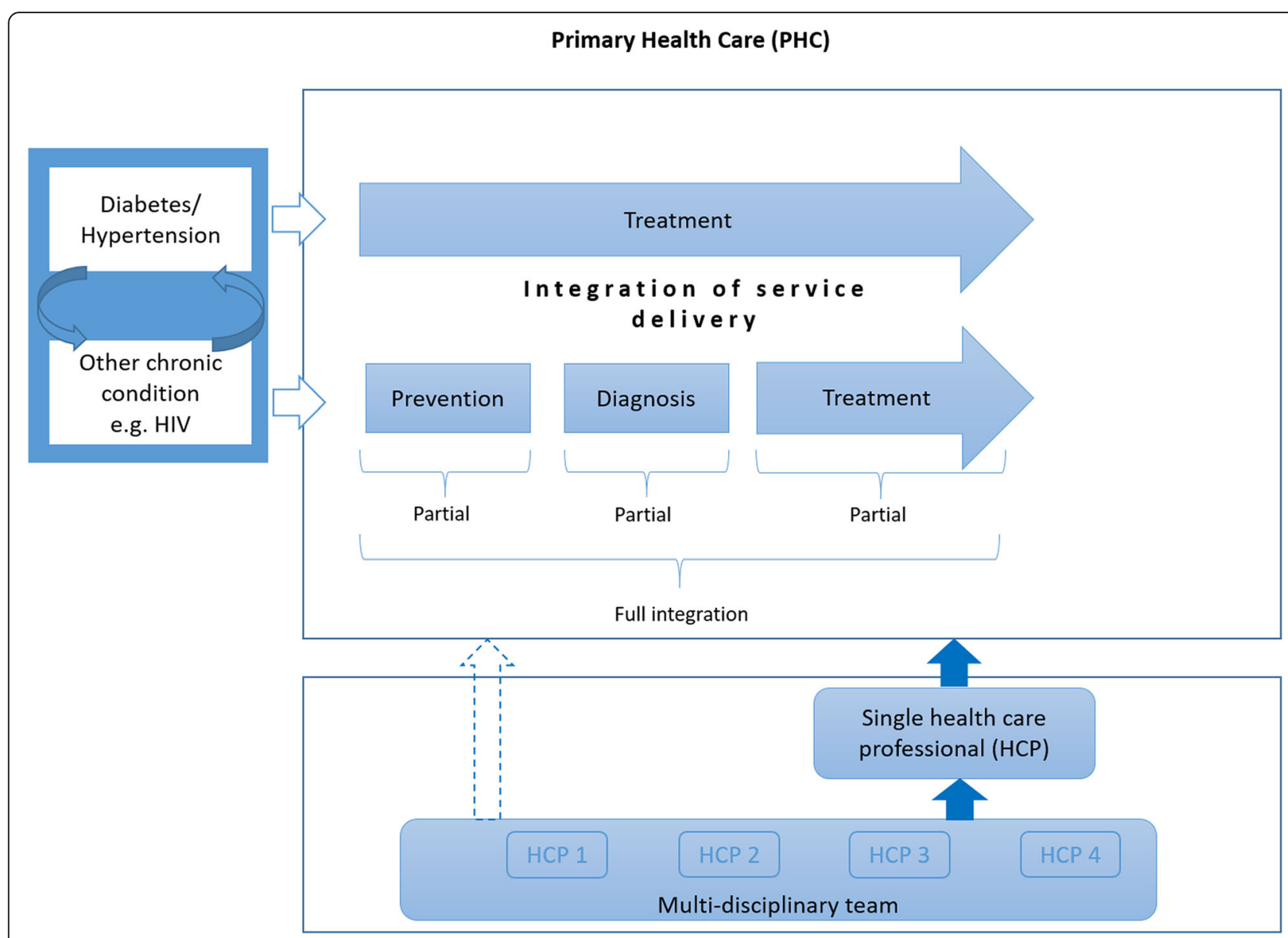

Fig. 1 Logic model of models of integrated care for diabetes and hypertension

There is thus little up-to-date evidence from LMICs on the effectiveness of integrated healthcare delivery for people with NCDs, and diabetes and hypertension in particular. With a rising burden of diabetes and hypertension, in combination with the existing high burden of infectious diseases and resource constrained health systems in LMICs, there is a need to optimise healthcare delivery to benefit both the patients and the health systems.

The objective of this systematic review is to assess the effects of integrated models of care at PHC level for people living in LMICs, with multi-morbidity, of which diabetes or hypertension is one, compared to no integrated care on health and process outcomes.

\section{Methods}

This protocol is reported according to the PRISMA-P checklist [32] (Additional file 1).

\section{Criteria for considering studies for the review}

- Types of participants
We will include studies examining people with multi-morbidity, of which diabetes and/or hypertension is one, living in LMICs, irrespective of age. We define multi-morbidity as the presence of two or more chronic medical conditions in an individual [8]. Studies addressing both adults and children will be considered. LMICs will be defined according to the classification of the World Bank [33].

\section{- Types of interventions}

This review will only consider studies that describe integration of service delivery at PHC and community level. We will consider models of partial integration and full integration of service delivery (Fig. 1). Partial integration of service delivery will be defined as models where patients treated for diabetes, hypertension or any other chronic disease receive part of the package of care (prevention, diagnosis, treatment) for another disease. Full integration of service delivery will be defined as models where patients (primarily treated for diabetes, hypertension or any other disease) receive the full 
package of care (prevention, diagnosis and treatment) for diabetes/hypertension and any other chronic disease at the same point of care by one or more healthcare professionals.

\section{- Types of comparisons}

The main comparison will be stand-alone models of care, defined as models of care that are limited to one disease. We will include the following comparisons:

- Fully integrated models of care vs. stand-alone care

- Partially integrated models of care vs. stand-alone care

- Fully integrated models of care vs. partially integrated models of care

\section{- Types of outcomes}

We will include studies that report on either primary health outcomes or secondary outcomes. However, the absence of reporting the pre-specified health and process outcomes will not be a deciding factor for inclusion of studies. Types of outcomes to consider are the following:

\section{Primary outcomes (health outcomes)}

- All-cause mortality

- Disease specific morbidity as reported in included

studies (e.g. disease control metrics, quality of life)

- Glycated haemoglobin (HbA1c)

- Systolic blood pressure (BP)

- Cholesterol levels

\section{Secondary outcomes (process outcomes)}

- Access to care as reported in the included studies

- Retention in care and adherence as reported in the included studies

- Continuity of care as reported in the included studies

- Quality of care as reported in the included studies

- Cost of care as reported in the included studies

\section{- Types of studies}

We will consider randomised controlled trials (RCTs), including cluster RCTs, controlled (non-randomised) clinical trials (CCTs) or cluster trials, interrupted time series (ITS) studies with at least three data points before and after the intervention, and controlled before-and-after (CBA) studies for inclusion. Cluster randomised, cluster non-randomised or CBA studies will be included only if there are at least two intervention sites and two control sites. Cross-sectional studies, case series and case reports will be excluded.

\section{Search methods for identifying studies}

We will search the following electronic databases: MEDLINE (PubMed), EMBASE (Ovid), the Cochrane Central Register of Control Trials (CENTRAL), LILACS, AfricaWide Information (via EBSCO host), CINAHL, Web of Science (Core collection). For ongoing studies, we will search the following trial registries: WHO ICTRP and Clinicaltrials.gov. We will search conference abstracts from the International AIDS Society Online Resource Library, the HIV/AIDS Implementers' Meetings and the NCDs Alliance meetings.

Search terms will include 'diabetes', 'hypertension', 'comorbidities , 'integrated healthcare delivery', 'low- and middle-income countries' and their synonyms. The full search strategy for MEDLINE (PubMed) is provided in Additional file 2. We will adapt it for other electronic databases. We will report all search strategies in full in the final version of the review. In addition, we will screen reference lists of included studies and reference lists of relevant systematic reviews, and contact experts in the field and relevant organisations (e.g. NCD Alliance) for unpublished studies. All languages will be included.

\section{Study selection and data extraction}

\section{- Selection of studies}

Two authors will independently screen titles and abstracts of studies identified by the search using Covidence software, and we will retrieve full-text of all potentially eligible studies. Two authors will independently screen full texts for eligibility. Discrepancies in the selection process will be resolved through discussion or by consulting a third author. Studies will be classified as included, excluded or awaiting assessment. We will provide reasons for excluding studies. Studies only available as abstracts will be included in the general results of the review, but not in the analysis.

\section{- Data extraction}

We will extract data based on the description of various models of integrated care as illustrated in Fig. 1 with consideration of possible scenarios of various packages of care provided in terms of partial integration. A pre-specified, standardised and piloted data extraction form will be used. Two authors will independently extract data and compare the results. Discrepancies will be resolved by discussion or by consulting a third reviewer. We will contact study authors in case of missing data. We will extract data on the participants, intervention, comparisons, outcomes, setting, context and funding sources. 
Data items will be in line with recommendations from the template for intervention description and replication (TIDieR) [34] and the PRISMA-Complex Interventions (CI) extension checklist [35]. We will look at the following items:

- Provide name or a phrase to describe the intervention

- Rationale, theory or goal of the element essential to the intervention

- Materials used

- Procedures or processes used in the intervention

- Who provided-each category of the intervention provider

- Describe modes of delivery of the intervention

- Types of location where the intervention took place

- When and how much-number of times the

intervention was delivered

- Tailoring of the intervention

- How well the intervention was planned

- Replicability

For the 'implementation components', we will look at the following items:

- Clearly define the adoption, uptake or integration strategies

Integration strategies may include facilitators (distinct from intervention elements) such as attestations, financial incentives, periodic reports of findings, reminders, supplemental trainings or physical environmental changes.

Where there is insufficient detail reported in the study, we will contact the study investigators for clarity or more information on the study. Disagreements will be resolved first by discussion and then by consulting at third author for consensus.

\section{- Risk of bias assessment}

For randomised controlled trials, non-randomised trials and controlled before-after studies, we will use the tool proposed by the Effective Practice and Organisation of Care (EPOC) group [36]. We will assess the following nine domains as having 'low risk', 'high risk' or 'unclear risk' of bias: (1) random sequence generation, (2) allocation concealment, (3) baseline outcome measurements, (4) baseline characteristics, (5) incomplete outcome data, (6) knowledge of allocated intervention (blinding), (7) protection against contamination, (8) selective outcome reporting and (9) other risks of bias. For cluster RCTs, we will assess additional risk of bias linked to recruitment, baseline differences, loss of clusters, incorrect analysis and compatibility with RCTs randomised by individuals. For ITS studies, we will also use the tool proposed by EPOC to assess whether: (1) the intervention was independent of other changes, (2) the shape of the intervention effect was pre-specified, (3) the intervention was unlikely to affect data collections, (4) knowledge of the allocated intervention was adequately prevented during the study, (5) incomplete outcome data was likely to bias results, (6) outcomes were reported selectively and (7) there were any other risks of bias. For each domain, risk of bias will be assessed as low, high or unclear. Two authors will independently assess risk of bias of included studies. We will resolve discrepancies through discussion or consulting a third author.

\section{Data analysis}

\section{- Measures of treatment effect}

We will extract relevant outcome data for each study and enter it into Review Manager 5. For dichotomous outcomes, we will calculate risk ratios (RR) and report pooled effects with $95 \%$ confidence intervals. For continuous outcomes, we will calculate the mean differences (MD) if outcomes were measured in the same way across studies or standardised mean differences (SMD) where outcomes were measured differently across studies. We will report pooled effects with $95 \%$ confidence intervals.

\section{- Unit of analysis issues}

Where cluster RCTs have appropriately adjusted for the effects of clustering in their analysis, we will use these adjusted effect estimates and standard errors in our meta-analysis using the generic inverse-variance method in Review Manager 5 [37]. Where the included cluster RCTs did not perform any adjustment for clustering, we will adjust the raw data ourselves using the intra-class correlation coefficient (ICC). If the study authors do not report an ICC value in the published article, either we will obtain this value from similar studies or we will estimate the ICC value. We will not present results from cluster RCTs that were not adjusted for clustering. If we estimate the ICC value, we will perform sensitivity analyses to investigate the robustness of our analyses. Where multi-arm studies (e.g. two intervention arms and one control arm) contribute multiple comparisons to a specific analysis, we will split the 'shared group' to avoid including data from the same participant more than once.

\section{- Dealing with missing data}

We will contact the study authors to request missing data if needed. If after contacting the authors, there are still missing data and we consider the data to be missing at random, we will include only the data available in the 
analysis. Otherwise, we will impute the missing data and account for the data imputed with uncertainty in line with the Cochrane Handbook of Systematic Reviews of Interventions [38]. We will then conduct a sensitivity analysis to analyse how sensitive the results are to the assumptions we made when imputing missing data and analyse all data as intention-to-treat.

\section{- Assessment of heterogeneity}

We will explore clinical heterogeneity by clearly documenting study characteristics related to the population, intervention, outcomes and context in table format. We will assess the statistical heterogeneity in each meta-analysis by inspecting forest plots and calculating $X^{2}$ test values and $I^{2}$ statistics. We will consider significant heterogeneity present if the $P$ value of the $\chi^{2}$ test is $<0.10$. We will interpret the $I^{2}$ statistic according to the thresholds recommended in the Cochrane Handbook of Systematic Reviews of Interventions [39]. Therefore, we will consider an $I^{2}$ value above 30\% to indicate important heterogeneity. In addition, we will explore the causes of statistical heterogeneity by conducting subgroup analyses.

\section{- Assessment of reporting biases}

We will examine reporting biases by means of funnel plots, if we are able to pool more than 10 studies per outcome in a meta-analysis.

\section{- Data synthesis}

We will organise the review findings according to the proposed models of integrated care as depicted in Fig. 1. We will pool data from individual studies if they are sufficiently homogeneous in terms of design, population, intervention and comparator. As we anticipate some degree of heterogeneity, we will perform random-effects meta-analysis. We will not pool data from RCTs and non-randomised studies in a single meta-analysis. If we judge included studies to be too heterogeneous to pool, we will make use of narrative synthesis and present data in table format.

\section{- Subgroup analysis}

We will carry out the following subgroup analyses on primary outcomes to explore heterogeneity: various co-morbidities (e.g. patients with diabetes and HIV vs patients with diabetes and depression), clinic vs. community level and age category (children, age 1-10 years vs. adolescents, age 10 to 19 years vs. adults, age $>19$ years).

\section{- Sensitivity analysis}

We will carry out the following sensitivity analyses on primary outcomes:

- To examine the effect of excluding studies of high risk of bias

- To examine the effect of various ICCs in case of adjusting outcomes for clustering ourselves

- To examine the effect of imputed data

\section{- Summary of findings table and certainty of evidence}

We will assess the certainty of the evidence for primary outcomes (all-cause mortality, disease-specific morbidity, HbA1c, BP, cholesterol levels) using GRADE. We will create a 'summary of findings' table using GRADEpro software [40]. In the table, we will display the model of integrated care, primary outcomes (e.g. all-cause mortality, disease-specific morbidity) of the review, the comparative risks between intervention and control groups, the relative effects with $95 \% \mathrm{CI}$, the number of participants in the studies and the certainty of evidence.

The five domains that we will consider for our judgement to downgrade the certainty of evidence comprise study limitations, inconsistency, imprecision, indirectness and publication bias. We will consider upgrading the certainty of evidence if there is a large effect, a dose-response and cases where all plausible residual confounding would reduce a demonstrated effect or would suggest a spurious effect if no effect was observed. The quality of evidence for each outcome will be described as high, moderate, low or very low [41].

\section{Discussion}

Given the rising burden of NCDs in light of communicable diseases in LMICs, policy-makers and healthcare professionals are seeking ways to provide comprehensive care to people with multi-morbidity. However, there is a lack of evidence on the effectiveness of integrated models of care in LMICs, hindering implementation of such models. Our systematic review aims to address this gap in evidence by providing an up-to-date and comprehensive synthesis of existing research. We will ensure methodological rigour by conducting an extensive search, systematically screening and selecting studies, extracting data and assessing the risk of bias of included studies. Furthermore, we will use GRADE to assess the quality of evidence. We have been engaging with policy-makers from the onset of this protocol, have involved them in the process of defining and clarifying the review question. We are also planning to continue this engagement throughout the review process. Hence, we believe that the findings of this systematic review will be relevant to policy-makers and inform policy and practice in LMICs. 


\section{Additional files}

Additional file 1: PRISMA-P checklist. (PDF $159 \mathrm{~kb}$ )

Additional file 2: Search strategy for MEDLINE (PubMed). (PDF 103 kb)

\section{Abbreviations}

ART: Antiretroviral treatment; BP: Blood pressure; CBA: Controlled before-andafter; CCT: Controlled clinical trials; CDs: Communicable diseases; CEBHA +: Collaboration for evidence Based Healthcare and Public Health in Africa; CENTRAL: The Cochrane Central Register of Control Trials; CINHAL: Cumulative Index to Nursing and Allied Health Literature: EMBASE: Excerpta Medica Database; EPOC: Effective Practice and Organisation of Care; GRADE: Grading of Recommendations Assessment, Development and Evaluation; HbA1c: Glycated haemoglobin; HIV: Human Immuno-deficiency Virus; ICC: Intra-class correlation coefficient; ITS: Interrupted time series; LILACS: Literatura Latino-Americana e do Caribe em Ciênciasda Saúde; LMICs: Low- and middle income countries; MD: Mean difference; MEDLINE: Medical Literature Analysis and Retrieval System Online; NCDs: Non-communicable diseases; PHC: Primary healthcare; PLWH: People living with HIV; PRISMA: The Preferred Reporting Items for Systematic Reviews and Meta-Analyses; RCTs: Randomised controlled trials; RR: Risk ratio; SMD: Standardised mean difference; TB: Tuberculosis; TIDieR: Template for Interventions Description and Replication; WHO: World Health Organisation

\section{Acknowledgements}

The authors would like to thank Mrs. Sandhya Singh and Mrs. Shaidah Asmall from the National Department of Health, South Africa for their inputs on the systematic review question.

\section{Funding}

The review work was supported by the funding from the Collaboration for Evidence-based Healthcare and Public Health in Africa (CEBHA+) project which is funded by the German Federal Ministry of Education and Research (BMBF) as part of the Research Networks for Health Innovation in sub-Saharan Africa Funding Initiative. The funder does not have any role in the review process

\section{Availability of data and materials} N/A

\section{Authors' contributions}

JUN, AR and TY drafted the protocol, critically revised it and approved the final version. $C M B$ contributed in the general conception of the review protocol, critically revised and approved the final version. JJM provided methodological input, critically revised manuscript, read and approved final version.

\section{Authors' information}

JUN and AR are senior researchers at the Centre for Evidence-based Healthcare (CEBHC), Division of Epidemiology and Biostatistics, Department of Global Health, Stellenbosch University. TY is the director of the CEBHC and head of Division of Epidemiology and Biostatistics, Department of Global Health, Stellenbosch University. CMB is clinician and lecturer at the College of Medicine and Health Sciences, University of Rwanda. JJM is the director of GRADE Centre Freiburg and co-director of Cochrane Germany.

\section{Ethics approval and consent to participate}

Since the current systematic review and meta-analysis will not involve any human participants, no ethics approval is needed as well as the consent to participate.

\section{Consent for publication}

Not applicable

\section{Competing interests}

The authors declare that they have no competing interests.

\section{Publisher's Note}

Springer Nature remains neutral with regard to jurisdictional claims in published maps and institutional affiliations.

\section{Author details}

${ }^{1}$ Centre for Evidence-Based Health Care, Division of Epidemiology and Biostatistics, Department of Global Health, Faculty of Medicine and Health Sciences, Stellenbosch University, Francie van Zijl drive, Parow, Cape Town 7500, South Africa. ${ }^{2}$ School of Public Health, College of Medicine and Health Science, University of Rwanda, Kicukiro, Kigali, Rwanda. ${ }^{3}$ College of Medicine and Health Science, University of Rwanda, Kicukiro, Kigali, Rwanda. ${ }^{4}$ Institute for Evidence in Medicine (for Cochrane Germany Foundation), Medical Center-University of Freiburg, Breisacher Strasse 153, 79110 Freiburg, Germany.

Received: 2 August 2018 Accepted: 31 October 2018

Published online: 20 November 2018

\section{References}

1. WHO. Non-Communicable diseases factsheet, 2017. http://www.who.int/ mediacentre/factsheets/fs355/en/, Accessed 13 Mar 2018.

2. GBD 2016 Disease and Injury Incidence and Prevalence Collaborators. Global, regional, and national incidence, prevalence, and years lived with disability for 328 diseases and injuries for 195 countries, 1990-2016: a systematic analysis for the Global Burden of Disease Study 2017. Lancet. 2017;390:1211-59.

3. NCD Alliance. Tackling non-communicable diseases in workplace settings in low- and middle-income countries. A CALL TO ACTION AND PRACTICAL GUIDANCE. Non- Communicable diseases (NCD) Alliance, 2017. www. ncdalliance.org. Accessed 13 Mar 2018.

4. Yusuf S, Wood D, Ralston J, Reddy K. The world heart federation's vision for worldwide cardiovascular disease prevention. Lancet. 2015;386(9991):399-402.

5. IDF diabetes Atlas 8th edition. https://www.idf.org/e-library/epidemiologyresearch/diabetes-atlas/134-idf-diabetes-atlas-8th-edition.html. Accessed 14 Mar 2018

6. Campbell NR, Lackland DT, Niebylski ML. High blood pressure: why prevention and control are urgent and important: a 2014 fact sheet from the World Hypertension League and the International Society of Hypertension. J Clin Hypertens (Greenwich). 2014:16(8):551-3. https://doi. org/10.1111/jch.12372

7. Wallace E, Guthrie B, Fahey B. Managing patients with multi-morbidity in primary care. BMJ. 2015;350:h176 doi: https://doi.org/10.1136/bmj.h176.

8. Lee GM, Jeong HM, Lee HK, Park HK, Sim SD, Yoon JH, et al. Prognostic impact of diabetes mellitus and hypertension for mid-term outcome of patients with acute myocardial infarction who underwent percutaneous coronary intervention. J Cardiol. 2012:60(4):257-63.

9. Barr AL, Young EH, Smeeth L, Newton R, Seeley J, Ripullone K, et al. The need for an integrated approach for chronic disease research and care in Africa. Global Health Epidemiol Genomics. 2016:e19 https://doi.org/10.1017/ gheg.2016.16.

10. Lalkhen $\mathrm{H}$, Mash B. Multi-morbidity in non-communicable diseases in south African primary healthcare. S Afr Med J. 2015;105(2):134-8. https://doi.org/ 10.7196/SAMJ.8696.

11. Remais VJ, Zeng G, Li G, Tian L, Engelgau MM. Convergence of noncommunicable and infectious diseases in low- and middle-income countries. Int J Epidemiol. 2013:42:221-7. https://doi.org/10.1093/ije/dys135.

12. UNAIDS. Chronic care for HIV and non-communicable diseases: how to leverage the HIV experience. Geneva: UNAIDS; 2011. http://www.unaids.org/ sites/default/files/media_asset/20110526_JC2145_Chronic_care_of_HIV_0. pdf. Accessed 16 Mar 2018

13. Nigatu T. Integration of HIV and Non-communicable Diseases in Health Care Delivery in Low- and Middle-Income Countries. Prev Chronic Dis. 2012 9:110331 doi: https://doi.org/10.5888/pcd9.110331.

14. Collaborators $\mathrm{GH}$. Estimates of global, regional, and national incidence, prevalence, and mortality of HIV, 1980-2015: the global burden of disease study 2015. Lancet HIV. 2016;3(8):e361-87.

15. Legido-Quigley H, Montgomery MC, Khan P, Atun R, Fakoya A, Getahun H, Grant AD. Integrating tuberculosis and HIV services in low- and middleincome countries: a systematic review. Trop Med Int Health. 2013:18(2):199_ 211. https://doi.org/10.1111/tmi.12029. 
16. Atlantis E, Fahey P, Foster J. Collaborative care for comorbid depression and diabetes: a systematic review and meta-analysis. BMJ Open. 2014;4:e004706. https://doi.org/10.1136/bmjopen-2013-004706.

17. Huang $Y$, Wei $X$, Wu T, Chen R, Guo A. Collaborative care for patients with depression and diabetes mellitus: a systematic review and meta-analysis. BMC Psychiatry. 2013;13:260. https://doi.org/10.1186/1471-244X-13-260.

18. Igwe MN, Uwakwe R, Ahanotu CA, Onyeama GM, Bakare MO, Ndukuba AC. Factors associated with depression and suicide among patients with diabetes mellitus and essential hypertension in a Nigerian teaching hospital. Afr Health Sci. 2013;13(1):68-77.

19. WHO. Package of essential non-communicable (PEN) disease interventions for primary health care in low-resource settings. Geneva: WHO; 2010. http:// www.who.int/nmh/publications/essential_ncd_interventions_Ir_settings.pdf. Accessed 15 Mar 2018

20. Mendis S, Al Bashir I, Dissanayake L, Varghese C, Fadhil I, Marhe E, et al. Gaps in capacity in primary care in low-resource settings for implementation of essential non-communicable disease interventions. Int J Hypertens. 2012;2:1-7. https://doi.org/10.1155/2012/584041.

21. Haldane H, Legido-Quigley H, Leh Hoon Chuah F, Sigfrid L, Murphy G, Ong ES, et al. Integrating cardiovascular diseases, hypertension, and diabetes with HIV services: a systematic review. AIDS Care. 2018;30(1):103-15. https:// doi.org/10.1080/09540121.2017.1344350.

22. Atun $\mathrm{R}$, de Jongh $\mathrm{T}$, Secci $\mathrm{F}$, Ohiri $\mathrm{K}$, Adeyi O. Integration of targeted health interventions into health systems: a conceptual framework for analysis. Health Policy Plan. 2010a;25:104-11.

23. Curry N, Ham C. Clinical and service integration: The route to improved outcomes. Int J Integr Care. 2012;12:e199. URN: NBN:NL:UI:10-1-113826.

24. Valentijn PP, Schepman MS, Opheij W, Bruijnzeels AM. Understanding integrated care: a comprehensive conceptual framework based on the integrative functions of primary care. Int J Integr Care. 2013;13:e010. JanMar, URN:NBN:NL:UI:10-1-114415.

25. Shigayeva A, Atun R, McKee M, Coker R. Health systems, communicable diseases and integration. Health Policy Plan. 2010;25:14-i20. https://doi.org/ 10.1093/heapol/czq060.

26. Atun R, de Jongh T, Secci F. A systematic review of the evidence on integration of targeted health interventions into health systems. Health Policy Plan. 2010b;25:1-14.

27. Naidoo K, Gengiah S, Yende-Zuma N, Padayatchi N, Barker P, et al. Addressing challenges in scaling up TB and HIV treatment integration in rural primary healthcare clinics in South Africa (SUTHI): a cluster randomized controlled trial protocol. Implement Sci. 2017;12(1):129. https://doi.org/10. 1186/s13012-017-0661-1.

28. Uyei J, Coetzee D, Macinko J, Guttmacher S. Integrated delivery of HIV and tuberculosis services in sub-Saharan Africa: a systematic review. Lancet Infect Dis. 2011;11(11):855-67. https://doi.org/10.1016/s1473-3099(11)70145-1.

29. Yiu KC, Rohwer A, Young T. Integration of care for hypertension and diabetes: a scoping review assessing the evidence from systematic reviews and evaluating reporting. BMC Health Serv Res. 2018;18:481 doi.org/10. 1186/s12913-018-3290-8

30. Janssens B, Van Damme W, Raleigh B, Gupta J, Khem S, Soy Ky K, Vun MC, Ford N, Zachariah R. Offering integrated care for HIV/AIDS, diabetes and hypertension within chronic disease clinics in Cambodia. Bull World Health Organ. 2007:85:880-5. https://doi.org/10.2471/BLT.06.036574.

31. Dudley L, Garner P. Strategies for integrating primary health services in lowand middle-income countries at the point of delivery. Cochrane Database Syst Rev. 2011;(7):CD003318. https://doi.org/10.1002/14651858.CD003318.pub3.

32. Moher D, Shamseer $L$, Clarke M, Ghersi D, Liberati A, Petticrew $M$ et al. Preferred reporting items for systematic review and meta-analysis protocols (PRISMA-P) 2015 statement. BMJ 2015; 4:1. dio: https://doi.org/10.1186/20464053-4-1

33. World Bank (2017). World Bank country and lending groups. Geneva: World Bank Group; 2017. datahelpdesk.worldbank.org/knowledgebase/articles/ 906519-world-bank-country-and-lending-groups. Accessed 14 Mar 2018

34. Hoffmann CT, Glasziou PP, Boutron I, Milne R, Perera R, Moher D, et al. Better reporting of interventions: template for intervention description and replication (TIDieR) checklist and guide. BMJ. 2014;348:g1687 doi: https://doi. org/10.1136/bmj.g1687.

35. Guise MJ, Em B, Chang C, Viswanathan M, Pigott T, Tugwell P, Complex Interventions Group. AHRQ series on complex intervention systematic reviews- papers6: PRISMA-Cl extension statement and checklist. J Clin Epidemiol. 2017;90:43-50. https://doi.org/10.1016/j.jclinepi.2017.06.016.
36. Cochrane Effective Practice and Organisation of Care (EPOC). Suggested risk of bias criteria for EPOC reviews. EPOC Resources for review authors. http:// epoc.cochrane.org/resources/epoc-resources-review-authors. Accessed 14 Mar 2018

37. RevMan [Computer program]. Nordic Cochrane Centre, The Cochrane Collaboration. Review Manager 5 (RevMan 5). Version 5.3. Copenhagen: Nordic Cochrane Centre, The Cochrane Collaboration; 2014.

38. JPT H, Green S. Cochrane handbook for systematic interventions version 5.1. O(updated March 2011): The Cochrane Collaboration; 2011. Available from https://training.cochrane.org/handbook

39. GRADEpro GDT. McMaster University (developed by Evidence Prime). GRADEpro GDT. Hamilton: Mc Master University; 2015. www.gradepro.org

40. Balshem H, Helfand M, Schunemann HJ, Oxman AD, Kunz R, Brozek J, et al. GRADE guidelines: 3. Rating the quality of evidence. J Clin Epidemiol. 2011; 64(4):401-6.

41. Guyatt G, Oxman AD, Akl EA, Kunz R, et al. GRADE guidelines: 1. IntroductionGRADE evidence profiles and summary of findings tables. J Clin Epidemiol. 2011;64(4):383-94
Ready to submit your research? Choose BMC and benefit from:

- fast, convenient online submission

- thorough peer review by experienced researchers in your field

- rapid publication on acceptance

- support for research data, including large and complex data types

- gold Open Access which fosters wider collaboration and increased citations

- maximum visibility for your research: over $100 \mathrm{M}$ website views per year

At $\mathrm{BMC}$, research is always in progress.

Learn more biomedcentral.com/submissions 